
\title{
R Research S Surare \\ IncRNA HOXA-AS2 functions as an oncogene by binding to EZH2 and suppressing LATS2 in Acute myeloid leukemia (AML)
}

\section{Yubin Feng}

Anhui Medical University

Shuang Hu

Anhui Medical University

Lanlan Li

Anhui Medical University

Xiaoqing Peng

Anhui Medical University

Feihu Chen ( $\nabla$ feng1876102@163.com )

Anhui Medical University

\section{Research}

Keywords: long non-coding RNAs, acute myeloid leukemia (AML), LATS2, ZEH2, HOXA-AS2, proliferation, differentiation

Posted Date: September 8th, 2020

DOl: https://doi.org/10.21203/rs.3.rs-70829/v1

License: (c) (i) This work is licensed under a Creative Commons Attribution 4.0 International License. Read Full License 


\section{Abstract}

\section{Background}

Long noncoding RNAs (IncRNAs) plays an important role in the development of physiology and pathology. Many reports have shown that IncRNA HOXA cluster antisense RNA 2 (HOXA-AS2) is a carcinogen and plays an important role in many tumors, but little is known about its role in Acute myeloid leukemia (AML).

\section{Methods}

The expression of HOXA-AS2 in AML cell line was detected by qRT-PCR. AML cases from the public database (GEPIA) were also included in this study. Cell counting kit-8 (CCK-8) assay, flow cytometry, immunofluorescence and Western blot were used to detect the role of HOXA-AS2 in AML cells. Luciferase reporter gene detection, RIP, RNA pull-down and RNA-ChIP detection were used to demonstrate the molecular biological mechanism of HOXA-AS2 in AML.

Results

HOXA-AS2 was upregulated in AML cell lines and tissues, and the overexpression of HOXA-AS2 is negatively correlated with the survival of patients. Silencing HOXA-AS2 can inhibit the proliferation and induce differentiation of AML cells in vitro and in vivo. Overexpressing HOXA-AS2 showed the opposite result. Moreover, more in-depth mechanism studies showed that carcinogenicity of HOXA-AS2 exerted mainly through binding with the epigenetic inhibitor Enhancer of zeste homolog 2 (EZH2) and then inhibiting the expression of Large Tumor Suppressor 2 (LATS2).

\section{Conclusions}

Taken together, our findings highlight the important role of HOXA-AS2 in AML, suggesting that HOXA-AS2 may be an effective therapeutic target for patients with AML.

\section{Background}

The two main features of AML are uncontrolled malignant proliferation and blocked differentiation, accounting for $30 \%$ of leukemia related deaths $[8,27]$. With the development of science and technology, the treatment of leukemia has been made great progress, but the cure of the disease is still unsolved and the five-year survival rate is only $25 \%$. Moreover, the risk of relapse and drug resistance will still occur during chemotherapy, which may become the dilemma of $\operatorname{AML}$ treatment $[4,10]$.

According to the results of human genome research, only a small part of human genes can encode protein, while the remaining large part of human genome genes $(\sim 97 \%)$ are tightly transcribed into noncoding RNA $[9,22,24]$. As a new noncoding RNA molecule, the abnormal expression of IncRNA in tumor tissue has attracted extensive attention of researchers $[23,28]$. Many studies have shown that 
IncRNA is likely to be a carcinogen gene $[18,19]$ or a tumor suppressor gene $[18]$ in the occurrence and development of tumors, and has increasingly become a biomarker of tumor diagnosis, treatment or prognosis, including AML $[15,36]$. Therefore, it is very important to identify more new cancer related IncRNAs and explore their biological functions and molecular mechanisms in order to explore new therapeutic methods of AML.

As a 1048-bp IncRNA, HOXA-AS2 has been shown to enhance gastric cancer proliferation through epigenetic erythrocyte sedimentation rate (ESR) silencing the expression of p21, plk3 and DDIT3 [37, 40]. However, little is known about the role of HOXA-AS2 in the pathogenesis and development of AML. Our results showed that HOXA-AS2 was up-regulated in AML cells. TCGA database also showed that HOXAAS2 expression was also up-regulated in AML tissues and was associated with poor prognosis in AML patients. Moreover, silencing of HOXA-AS2 not only inhibited the proliferation and induced differentiation of AML cells, but also reduced the tumorigenicity of nude mice. We further proved that HOXA-AS2 acts as a modular scaffold for histone modified complexes by binding with EZH2 to silence LATS2 expression. In conclusion, our results suggest that HOXA-AS2-EZH2-LATS2 axis may provide a new strategy for the diagnosis and treatment of AML.

\section{Materials And Methods}

\section{Cell Lines and Culture}

NB4 and THP-1 cell lines were provided by Shanghai Gecko gene in 1640 medium (Gibco, Grand Island, NY, USA) containing $10 \%$ fetal bovine serum (FBS) (Gibco, Grand Island, NY, USA) containing penicillin $(100 \mathrm{U} / \mathrm{ml})$ and streptomycin $(100 \mathrm{~g} / \mathrm{ml})$ (Gibco, Grand Island, NY, USA). All cells were cultured in a humidified atmosphere, at $37{ }^{\circ} \mathrm{C}$ with $5 \% \mathrm{CO}_{2}$. We changed the cell culture medium every 24 hours, and also subcultured the cells for 48 hours.

\section{RNA extraction and qRT-PCR assays}

Trizol (Invitrogen, USA) was used to extract total RNA from tissues and cell lines, and then reverse transcripted using Primer-Script One Step RTPCR kit (TaKaRa, China). Finally, SYBR premix dimming eraser Kit (TaKaRa, China) was used for real-time RT-PCR detection. GAPDH was used as a standardized control. All measurements were repeated three times. The primer sequences are shown in Table 1.

\section{Gene knockdown}

Small interfering RNA (siRNA) and short hairpin RNA (shRNA) were used for knockdown of genes. Lipofectamine 2000 (Invitrogen) was used for transient transfection, and the correlation was performed 48 hours after transfection. We purchased HOXA-AS2 siRNA (siHOXA-AS2) and negative control siRNA (siNC) from Bioujing Bio (Shanghai, China). The primer interference sequences are shown in Table 1. 
HOXA-AS2 short-hairpin RNA (LV-shHOXA-AS2) and respective negative control (LV-shNC) were obtained from Bioujing Bio (Shanghai, China). First, NB4 and THP-1 cells were seeded in 24-well plates before being transfected. Then, $30 \mathrm{ml}$ shRNA was added to each well, allowed to stand at room temperature for $15 \mathrm{~min}$, and placed in a cell culture incubator; and the medium was changed after $24 \mathrm{~h}$. The primer interference sequences are shown in Table 1.

\section{Gene overexpression}

pcDNA-LATS2 vector and pcDNA3.1 vector, as well as pCMV6-XL5-HOXA-AS2 were generous gift from ZSP (Wannan Medical College). Amplification efficiencies were determined by qRT-PCR.

\section{Cell counting kit-8 (CCK-8) assays}

CCK-8 kit was used for detection (Beyotime Institute of Biotechnology, China). The transformed cells were inoculated into 96 well plate $\left(1 \times 10^{3}\right.$ cells/well), and then detected at $450 \mathrm{~nm}$ absorbance every 24 hours for 96 hours. Five multiple holes were taken for testing each time, and all the assays were conducted in triplicate.

\section{Cell Cycle Analysis}

The transfected cells were collected and fixed with $70 \%$ precooled ethanol. Then according to the instructions of Shanghai Beibo biological cell cycle detection kit. Finally, cell cycle was detected by CytoFLEX (Becton Dickinson, USA) and analyzed by ModFit software.

\section{Differentiation Marker Analysis}

The detection methods of differentiation indicators (CD11b and CD14) has been presented in our previously published articles [11].

\section{Western blotting}

We first used $8 \%$ SDS-PAGE to isolate the protein lysate, and then transferred the protein to 0.22um PDVF membrane. PDVF membrane was incubated with the corresponding primary antibody at $4{ }^{\circ} \mathrm{C}$ overnight, and then incubated with horseradish peroxidase labeled secondary antibody at room temperature. All the experiments were repeated three times. $\beta$-actin (Cat No. bsm-33036M, Bioss, Beijing, China), rabbit antihuman LATS2 (Cat No. 5888, CST, Boston, United States), rabbit anti-human CDK4 (Cat No. ab108357, Abcam, Danvers, MA, United States), rabbit anti-human Cyclin A2 (Cat No. ab181591, Abcam, Danvers, MA, United States), rabbit anti-human Cyclin D3 (Cat No. ab183338, Abcam, Danvers, MA, United States), rabbit anti-human P-Rb (Cat No. ab184796, Abcam, Danvers, MA, United States), rabbit anti-human CD11b (Cat No. ab133357, Abcam, Danvers, MA, United States), rabbit anti-human CD14 (Cat No. ab133335, Abcam, Danvers, MA, United States), and rabbit anti-human EZH2 (Cat No. ab186006, Abcam, Danvers, MA, United States). 


\section{RNA immunoprecipitation (RIP)}

The treated cells were collected and their nuclear proteins were extracted and then resuspended in RIP buffer. Then divide the resuspended RIP buffer into input group, IgG group and EZH2 group. Then collect the supernatant after centrifugation, add IgG (Abcam) or human anti-EZH2 antibody (Abcam), and then incubate at $4^{\circ} \mathrm{C}$ for 2 hours. Subsequently, protein $\mathrm{A}$ beads were added and incubated at $4^{\circ} \mathrm{C}$ for 1 hour. After centrifugation, the cells were washed 3 times with RIP buffer and then once with PBS, and the beads were resuspended in Trizol. Finally, quantitative detection was performed by reverse transcription quantitative polymerase chain reaction (RT-qPCR).

\section{Luciferase Reporter Assay}

Human LATS2 luciferase reporter gene plasmid was prepared and synthesized by Bioujing bio. The luciferase activities of firefly and marine kidney were measured (Promega, Shanghai, China) consecutively $40 \sim 44 \mathrm{~h}$ after transfection. Finally, the luciferase activity of firefly was quantified according to the luciferase standardization.

\section{RNA Pull-Down Assay}

Biotin-labeled RNA pull-down was performed as described previously (Xiang et al., 2014). In brief, $1 \times 10^{7}$ AML cells were harvested, lysed, and sonicated. Then, nuclear proteins were extracted using a nuclearplasma-isolation extraction kit and incubated with biotin-labeled HOXA-AS2 truncated probes and streptavidin agarose beads (Invitrogen) at $4{ }^{\circ} \mathrm{C}$ overnight. After washing with the wash buffer, the protein was recovered and detected by Western blot.

\section{Chromatin Immunoprecipitation (ChIP)}

ChIP analysis was carried out according to the manufacturer of EZ-ChIP kit (Upstate Biotechnology, Lake Placid, NY, USA). The final results were determined by RT-qPCR. The primers were designed by the Premier Primer 5.0 software

GAPDH:

forward, 5'-GAAGGTGAAGGTCGGAGTC-3',

reverse, 5'-GAAGATGGTGATGGGATTTC-3';

$\mathrm{EZH} 2$ :

forward, 5'-ACTTGGCTTCCAGCACCCG-3',

reverse, 5'-CGCTGTAAGGGACGCCACTG-3';

Fluorescence in situ Hybridization (FISH) 
Fluorescence in situ hybridization (FISH) was used to detect the location of HOXA-AS2 in the cells. The experimental method was similar to that reported before [11]. The cells were first fixed with $4 \%$ paraformaldehyde, then apply the cells on the anti-dropping glass slides to make droplets, then fix and permeabilize the cells, then incubate with FISH probes, stain with DAPI, and finally mount the slides. Fluorescence imaging was analyzed by laser scanning confocal microscope. The probe sequence of HOXA-AS2 for the FISH analysis have been provided.

\section{S-5' CTGCCTTCCTTGGATGTGGTAGCCGTTTC 3'}

\section{HOXA-AS2-5' TAGGGAGGTGAGGTGATTCGTT 3'}

\section{Tumor Xenografts}

All animal care and experiments were carried out in accordance with the guidance of the National Institutes of Health and approved by the Ethics Committee of Anhui Medical University. We selected 4week-old NCG mice with severe immunodeficiency for tumor xenotransplantation experiments to study the effect of HOXA-AS2 on tumor proliferation and differentiation. In tumor growth assay in vivo, NB4 cells stably transfected with HOXA-AS2 short-hairpin RNA (LV-shHOXA-AS2) and respective negative control (LV-shNC) were subcutaneously injected into the upper back of the nude mice $\left(1 \times 10^{7}, 200 \mu \mathrm{l}\right)$. Eight weeks after inoculation, the cells were killed. Finally, mice were sacrificed, and subcutaneous tumor tissues were detected for tumor weight, WB and IHC staining.

\section{Immunohistochemistry}

Tumor tissue was fixed in $4 \%$ paraformaldehyde and embedded in paraffin. After sectioning, it was incubated with CD11b antibody (Bioss, China), CD14 antibody (Bioss, China), Ki67 antibody (Bioss, China) and LATS2 antibody (CST, USA) overnight at $4{ }^{\circ} \mathrm{C}$. Then they were incubated with the second antibody and stained with diaminobenzidine. Photographs were taken with a light microscope.

\section{Nitroblue tetrazolium (NBT) assay}

NB4 $\left(1 \times 10^{5}\right.$ cells $\left./ \mathrm{ml}\right)$ was inoculated in a 6 -well plate and treated with ATPR. A $10 \mu$ l aliquot of NBT solution, composed of $10 \mathrm{mg} / \mathrm{ml} \mathrm{NBT}$ (Sigma-Aldrich) and $2 \mathrm{ug} / \mathrm{ml}$ PMA (Sigma-Aldrich), was added to each well, and then cells were incubated for $30 \mathrm{~min}$ at $37^{\circ} \mathrm{C}$. The positive cells ratio was analysed by light light microscopy.

\section{Statistical Analysis}

Data were expressed as mean \pm standard deviation. One-way analysis of variance (ANOVA) and Duncan test were used for comparison between multiple groups. If the $p$-value was less than 0.05 , the difference is considered statistically significant. The experimental results were represented at least three independent experiments. 


\section{Results}

\section{HOXA-AS2 levels were increased in AML}

In order to explore the role of HOXA-AS2 in AML, we first used GEPIA database to analyze the expression of HOXA-AS2 in AML patients. The results showed that the expression of HOXA-AS2 in AML tissues (left bar) was significantly higher than that in normal tissues (right bar) (Fig. 1a) (http://gepia.cancerpku.cn/detail.php). Furthermore, Kaplan Meier survival analysis showed that the overall survival rate of AML patients with high expression of HOXA-AS2 $(n=53)$ was significantly lower than the patients with low expression of HOXA-AS2 ( $n=53$ ) (Fig. 1b). qRT-PCR results showed that HOXA-AS2 was highly expressed in many leukemia cell lines (KG-1, NB4, U937, HL-60, and THP-1 cells) compared to the normal human monocytes (Fig. 1c). Based on the above results, we conducted a series of in vitro and in vitro studies to determine the function of HOXA-AS2 in AML.

\section{HOXA-AS2 regulates AML cell proliferation}

In order to further explore the biological function of HOXA-AS2 in AML cells, we choose the NB4 and THP1 cell lines for further studies. The efficiency of silencing and overexpression was verified by qRT-PCR (Fig. 1d, e). si-HOXA-AS2 1\# and 2\# demonstrated the better silencing capacity. HOXA-AS2 expression was increased in AML cell lines compared with the negative control following transfection with pCMV6XL5-HOXA-AS2. Thus, we selected si-HOXA-AS2 1\# and 2\# and pCMV6-XL5-HOXA-AS2 in all following experiments. CCK8 results showed that silencing HOXA-AS2 could inhibit the proliferation of AML cells, but overexpression of HOXA-AS2 in AML cells had the opposite effect (Fig. 2a, b). Flow cytometry and Western blot were then used to demonstrate the above results. The results of flow cytometry showed that inhibition of HOXA-AS2 expression could induce GO / G1 cycle arrest of AML cells, thus inhibit the proliferation of AML cells. On the contrary, overexpression of HOXA-AS2 had the opposite effect in AML cells (Fig. 2c, d). Overexpression of HOXA-AS2 in AML cells can increase the expression of G0/G1 marker protein (cyclin D3, cyclin A2, P-Rb and CDK4) (Fig. 2e). In contrast, our results also showed that silencing HOXA-AS2 could induce the decrease of G0/G1 marker protein (Fig. 2f). In order to further confirm the effect of HOXA-AS2 on the proliferation of AML cells, we used immunofluorescence to analyze the expression of Ki67 in the transfected AML cells (Fig. 2g, h). Western blot showed that silencing HOXAAS2 inhibited Ki67 expression compared with that in control tumors (Fig. 2i). These results demonstrated that HOXA-AS2 expression levels were associated with AML proliferation.

\section{HOXA-AS2 regulates AML cell differentiation}

Our results have demonstrated that HOXA-AS2 can regulate the proliferation of AML. As we all known, AML is mainly caused by blocked differentiation. We used a series of experiments to prove the effect of HOXA-AS2 on differentiation. Many studies have shown that CD11b and CD14 are classic differentiation markers of leukemia. Flow cytometry showed that knockdown of HOXA-AS2 could induce the differentiation of AML cells (Fig. 3b). On the contrary, overexpression of HOXA-AS2 decreased the proportion of differentiated cells in AML cells compared with control cells (Fig. 3a). Subsequently, 
Western blotting showed that silencing HOXA-AS2 promoted the expression of CD11b and CD14 in AML cells (Fig. 3d), whereas overexpression of HOXA-AS2 resulted in decreased expression of CD11b and CD14 (Fig. 3c). The reduction of NBT showed that HOXA-AS2 knockdown significantly increased the NBT reduction in AML cells compared with NC group (Fig. 3e). In addition, shRNA knockdown of HOXA-AS2 could promote the expression of CD11b and CD14 (Fig. 3f). In conclusion, these data suggested that HOXA-AS2 can not only regulate the proliferation of AML, but also regulate the differentiation of AML.

\section{HOXA-AS2 regulated LATS2 transcription in AML Cells}

In order to study the role of LATS2 in AML, we next explored the relationship between HOXA-AS2 and LATS2. As shown in Figure 4a, silencing HOXA-AS2 significantly increased LATS2 protein levels compared with negative controls. On the contrary, overexpression of HOXA-AS2 significantly decreased the expression of LATS2 (Fig. 4b). The results of gene level showed that inhibition of HOXA-AS2 could promote the expression of LATS2 mRNA (Fig. 4c), and overexpression of HOXA-AS2 could inhibit the expression of LATS2 mRNA in AML cells (Fig. 4d). To further elucidate the specific mechanism of HOXAAS2-regulating LATS2 expression, we used a human LATS2 luciferase reporter gene plasmid for the luciferase reporter gene analysis. Knocking down of HOXA-AS2 can increase LATS2 promoter activity in AML cells (Fig. 4e), whereas overexpression of HOXA-AS2 had the opposite effect (Fig. 4f). These results suggested that the transcription of LATS2 in AML cells can be regulated by HOXA-AS2.

\section{HOXA-AS2 regulated transcriptional expression of LATS2 through recruitment of EZH2 in AML cells}

The expression of HOXA-AS2 was further confirmed by FISH. The nucleus was labeled with DAPI, and HOXA-AS2 was labeled with Cy3 and 18S rRNA (cytoplasmic positive). The results showed that HOXAAS2 was located in the nuclei of AML cells (Fig. 5a). The next CHIP analysis results showed that overexpression of HOXA-AS2 can improve its binding ability to EZH2 (Fig. 5b). Many literatures have shown that EZH2 may be a key regulator of AML, therefore we silenced EZH2 expression using EZH2 siRNA. RT-qPCR and western blot results showed that the expression of EZH2 and LATS2 was negatively correlated, and silencing EZH2 could promote the expression of LATS2 (Fig. 5c and d). Subsequently, RNA immunoprecipitation (RIP) analysis showed that EZH2 protein could bind to HOXA-AS2 (Fig. 5e). The direct interaction between EZH2 and HOXA-AS2 was further demonstrated by applying the total protein (Fig. $5 f$ ) to the HOXA-AS2 pull-down assay. Taken together, we concluded that HOXA-AS2 regulated transcriptional expression of LATS2 through recruitment of EZH2 in AML cells.

\section{LATS2 silencing potentially involves the oncogenic function of HOXA-AS2}

Furthermore, to validate whether HOXA-AS2 regulates AML cell proliferation by silencing LATS2 expression, rescue assays were performed. AML cells were co-transfected with si-HOXA-AS2 and siLATS2, and the CCK8 assay results indicated that co-transfection partially rescued si-HOXA-AS2damaged proliferation ability (Fig. 6a). In addition, the ki67 was determined by immunofluorescence staining analysis also confirmed this result (Figure $5 b$ ). Furthermore, flow cytometry analysis showed that the decreased LATS2 expression reversed the G0 / G1 arrest induced by HOXA-AS2 silencing (Fig. 6c). 
Moreover, the western blot results indicated that co-transfection increased the downregulated expression of cyclin D3, cyclin A2, P-Rb and CDK4 triggered by the knockdown of HOXA-AS2 (Fig. 6d). Flow cytometry analysis showed that the decreased LATS2 expression reversed the higher level of differentiation resulted from HOXA-AS2 silencing (Fig. 6e). Moreover, the western blot results indicated that co-transfection decreased the upregulated expression of CD11b and CD14 induced by the knockdown of HOXA-AS2 (Fig. 6f). These results indicated that the effect of HOXA-AS2 on AML partially involves targeting LATS2.

\section{Knockdown of HOXA-AS2 inhibits AML tumorigenesis in vivo}

We have studied the in vitro study of HOXA-AS2 in AML cells, and the biological function of HOXA-AS2 in vivo. To further study the carcinogenic effect of HOXA-AS2 in AML, the control cells and shRNA HOXAAS2 NB4 cells were injected into nude mice. Tumor volume was measured every week after injection, and the tumor tissue was removed 4 weeks later. The results showed that compared with the control group, silencing HOXA-AS2 significantly inhibited the tumor growth (Fig. 7a and b). The tumor weight of HOXAAS2 knockout group was significantly lower than that of control group (Fig. 7c). Moreover, RT-PCR analysis showed that the expression level of HOXA-AS2 in tumor was lower than that after shRNA transfection (Fig. 7d). In addition, immunohistochemistry and Western blot showed that HOXA-AS2 upregulated LATS2 protein (Fig. 7e and f). In conclusion, these data suggested that HOXA-AS2 may affect the proliferation and differentiation of AML tumors in vivo, as well as in vitro experiments.

\section{Discussion}

With the continuous improvement and progress of experimental technology, more and more new IncRNAs have been discovered by RNA sequencing technology, which play a main role in the occurrence and development of diseases and malignant progress $[2,25,38]$. Many studies have shown that many IncRNAs express differently in AML and can regulate their functions $[11,12,21,30]$. However, whether the function and molecular mechanism of many IncRNAs are associated with AML is still unclear.

HOXA-AS2, as a new 1048 bp IncRNA, was first reported to act as an inhibitor of apoptosis in NB4 promyelocytic leukemia cells treated with all trans retinoic acid [40], and has been reported to be associated with many types of malignant tumors[33]. Studies have shown that the high expression of HOXA-AS2 may be related to various biological processes of malignant tumors, such as apoptosis, invasion, migration, proliferation and so on $[7,32,35,39]$. We found that HOXA-AS2 is highly expressed in AML tissues and cell lines. The predicted results from the database website (GEPIA) showed that the expression level of HOXA-AS2 was negatively related to the survival time of AML patients. In the current study, we found that silencing HOXA-AS2 in AML cells inhibited cell proliferation and induced differentiation. Overexpression of HOXA-AS2 had the opposite biological function. In vivo experiments also showed that knockout of HOXA-AS2 can inhibit tumor growth.

As a highly conserved histone methyltransferase, $\mathrm{EZH} 2$ is a subunit of $\mathrm{PRC} 2$, which plays a regulatory role mainly by triggering $\mathrm{H} 3 \mathrm{~K} 27 \mathrm{me} 3$ trimethylation [reference]. It can inhibit the translation of many target 
genes and regulate cell cycle regulation, aging, cell proliferation, differentiation, apoptosis and tumorigenesis $[20,29]$. Many studies have shown that IncRNA binds with EZH2 to exert its biological functions by regulating the expression of downstream genes $[5,34]$. Our recent results showed that ATPR can up-regulate the expression of IncRNA NR-104098 and inhibit the differentiation and proliferation of AML [11]. The in-depth exploration revealed that IncRNA NR-104098 played a role by enhancing the binding of E2F1 to EZH2 promoter [11]. Our results indicated that HOXA-AS2 can directly bind to EZH2 and inhibit the proliferation and induce differentiation of AML by regulating the expression of LATS2.

As a confirmed tumor suppressor [31], LATS2 plays an anti-tumor role through different signaling pathways $[14,16,17]$. Our ChIP results confirmed that EZH2 can directly bind to LATS2 promoter region in AML to regulate the expression of XXX. In addition, further rescue experiments showed that HOXA-AS2 played its biological function through LATS2. Silence of LATS2 can reverse this behavior.

\section{Conclusions}

In conclusion, the up-regulation of HOXA-AS2 may be associated with the negative prognosis of AML patients. This study first reported the high expression of HOXA-AS2 in AML tissues and cells. Silencing HOXA-AS2 can inhibit the tumorigenesis of nude mice, inhibit its proliferation and induce its differentiation in AML cells. Moreover, HOXA-AS2 inhibited the expression of LATS2 by binding with EZH2. Our findings suggested that HOXA-AS2 may be a new target for AML therapy, and provide a new hope for the diagnosis and treatment of AML targeting IncRNA.

\section{Abbreviations}

HOXA-AS2: LncRNA HOXA cluster antisense RNA 2

LncRNAs: Long noncoding RNAs

AML: Acute myeloid leukemia

CCK-8: Cell counting kit-8

EZH2: Enhancer of zeste homolog 2

LATS2: Large Tumor Suppressor 2

shRNA: short hairpin RNA

siRNA: Small interfering RNA

FISH: Fluorescence in situ hybridization

qRT-PCR: Quantitative real-time polymerase chain reaction 
FBS: fetal bovine serum

\section{Declarations}

\section{Availability of data and materials}

The datasets used in the current study are available from the corresponding

author on reasonable request.

\section{Acknowledgements}

Not applicable.

\section{Funding}

This work was supported by Anhui Science and Technology Major Project (No. 17030801020); and Chen Feihu Central Support for Local-Provincial Translational Medicine Project in 2017 (2017zhyx31)

\section{Authors' contributions}

FYB designed and performed experiments, collected and analyzed the data, wrote the paper. HS provided the methodology. LLL performed some experiments and provided technical and material support. PXQ, CFH designed and organized the study, and analyzed the data.

\section{Ethics approval and consent to participate}

The animal experiments were approved by the Anhui Medical University.

\section{Consent for publication}

Not applicable.

\section{Competing interests}

The authors declare that they have no competing interests.

\section{References}

[1] A. Akalin, F.E. Garrett-Bakelman, M. Kormaksson, J. Busuttil, L. Zhang, I. Khrebtukova, T.A. Milne, Y. Huang, D. Biswas, J.L. Hess, C.D. Allis, R.G. Roeder, P.J. Valk, B. Lowenberg, R. Delwel, H.F. Fernandez, E. Paietta, M.S. Tallman, G.P. Schroth, C.E. Mason, A. Melnick, M.E. Figueroa, Base-pair resolution DNA methylation sequencing reveals profoundly divergent epigenetic landscapes in acute myeloid leukemia, PLoS Genet, 8 (2012) e1002781. 
[2] L.M. Barnhill, R.T. Williams, O. Cohen, Y. Kim, A. Batova, J.A. Mielke, K. Messer, M. Pu, L. Bao, A.L. Yu, M.B. Diccianni, High expression of CAl2, a 9p21-embedded long noncoding RNA, contributes to advancedstage neuroblastoma, Cancer Res, 74 (2014) 3753-3763.

[3] L. Bullinger, M. Ehrich, K. Dohner, R.F. Schlenk, H. Dohner, M.R. Nelson, D. van den Boom, Quantitative DNA methylation predicts survival in adult acute myeloid leukemia, Blood, 115 (2010) 636-642.

[4] A. Burnett, M. Wetzler, B. Lowenberg, Therapeutic advances in acute myeloid leukemia, J Clin Oncol, 29 (2011) 487-494.

[5] Q. Chen, J. Cai, Q. Wang, Y. Wang, M. Liu, J. Yang, J. Zhou, C. Kang, M. Li, C. Jiang, Long Noncoding RNA NEAT1, Regulated by the EGFR Pathway, Contributes to Glioblastoma Progression Through the WNT/beta-Catenin Pathway by Scaffolding EZH2, Clin Cancer Res, 24 (2018) 684-695.

[6] S. Deneberg, P. Guardiola, A. Lennartsson, Y. Qu, V. Gaidzik, O. Blanchet, M. Karimi, S. Bengtzen, H. Nahi, B. Uggla, U. Tidefelt, M. Hoglund, C. Paul, K. Ekwall, K. Dohner, S. Lehmann, Prognostic DNA methylation patterns in cytogenetically normal acute myeloid leukemia are predefined by stem cell chromatin marks, Blood, 118 (2011) 5573-5582.

[7] J. Ding, M. Xie, Y. Lian, Y. Zhu, P. Peng, J. Wang, L. Wang, K. Wang, Long noncoding RNA HOXA-AS2 represses P21 and KLF2 expression transcription by binding with EZH2, LSD1 in colorectal cancer, Oncogenesis, 6 (2017) e288.

[8] H. Dohner, D.J. Weisdorf, C.D. Bloomfield, Acute Myeloid Leukemia, N Engl J Med, 373 (2015) 11361152.

[9] M. Esteller, Non-coding RNAs in human disease, Nat Rev Genet, 12 (2011) 861-874.

[10] E.J. Feldman, Novel Therapeutics for Therapy-Related Acute Myeloid Leukemia: 2014, Clin Lymphoma Myeloma Leuk, 15 Suppl (2015) S91-93.

[11] Y. Feng, S. Hu, L. Li, S. Zhang, J. Liu, X. Xu, M. Zhang, T. Du, Y. Du, X. Peng, F. Chen, LncRNA NR104098 Inhibits AML Proliferation and Induces Differentiation Through Repressing EZH2 Transcription by Interacting With E2F1, Front Cell Dev Biol, 8 (2020) 142.

[12] T.R. Fernando, J.R. Contreras, M. Zampini, N.I. Rodriguez-Malave, M.O. Alberti, J. Anguiano, T.M. Tran, J.K. Palanichamy, J. Gajeton, N.M. Ung, C.J. Aros, E.V. Waters, D. Casero, G. Basso, M. Pigazzi, D.S. Rao, The IncRNA CASC15 regulates SOX4 expression in RUNX1-rearranged acute leukemia, Mol Cancer, 16 (2017) 126.

[13] M.E. Figueroa, O. Abdel-Wahab, C. Lu, P.S. Ward, J. Patel, A. Shih, Y. Li, N. Bhagwat, A. Vasanthakumar, H.F. Fernandez, M.S. Tallman, Z. Sun, K. Wolniak, J.K. Peeters, W. Liu, S.E. Choe, V.R. Fantin, E. Paietta, B. Lowenberg, J.D. Licht, L.A. Godley, R. Delwel, P.J. Valk, C.B. Thompson, R.L. Levine, A. 
Melnick, Leukemic IDH1 and IDH2 mutations result in a hypermethylation phenotype, disrupt TET2 function, and impair hematopoietic differentiation, Cancer Cell, 18 (2010) 553-567.

[14] Y. Gao, J. Yi, K. Zhang, F. Bai, B. Feng, R. Wang, X. Chu, L. Chen, H. Song, Downregulation of MiR-31 stimulates expression of LATS2 via the hippo pathway and promotes epithelial-mesenchymal transition in esophageal squamous cell carcinoma, J Exp Clin Cancer Res, 36 (2017) 161.

[15] M. Gourvest, P. Brousset, M. Bousquet, Long Noncoding RNAs in Acute Myeloid Leukemia: Functional Characterization and Clinical Relevance, Cancers (Basel), 11 (2019).

[16] Y. Guo, J. Cui, Z. Ji, C. Cheng, K. Zhang, C. Zhang, M. Chu, Q. Zhao, Z. Yu, Y. Zhang, Y.X. Fang, W.Q. Gao, H.H. Zhu, miR-302/367/LATS2/YAP pathway is essential for prostate tumor-propagating cells and promotes the development of castration resistance, Oncogene, 36 (2017) 6336-6347.

[17] L. Hoa, Y. Kulaberoglu, R. Gundogdu, D. Cook, M. Mavis, M. Gomez, V. Gomez, A. Hergovich, The characterisation of LATS2 kinase regulation in Hippo-YAP signalling, Cell Signal, 28 (2016) 488-497.

[18] Y. Hu, J. Wang, J. Qian, X. Kong, J. Tang, Y. Wang, H. Chen, J. Hong, W. Zou, Y. Chen, J. Xu, J.Y. Fang, Long noncoding RNA GAPLINC regulates CD44-dependent cell invasiveness and associates with poor prognosis of gastric cancer, Cancer Res, 74 (2014) 6890-6902.

[19] J. Huang, N. Zhou, K. Watabe, Z. Lu, F. Wu, M. Xu, Y.Y. Mo, Long non-coding RNA UCA1 promotes breast tumor growth by suppression of p27 (Kip1), Cell Death Dis, 5 (2014) e1008.

[20] P. Joshi, E.A. Carrington, L. Wang, C.S. Ketel, E.L. Miller, R.S. Jones, J.A. Simon, Dominant alleles identify SET domain residues required for histone methyltransferase of Polycomb repressive complex 2, J Biol Chem, 283 (2008) 27757-27766.

[21] H. Luo, G. Zhu, J. Xu, Q. Lai, B. Yan, Y. Guo, T.K. Fung, B.B. Zeisig, Y. Cui, J. Zha, C. Cogle, F. Wang, B. Xu, F.C. Yang, W. Li, C.W.E. So, Y. Qiu, M. Xu, S. Huang, HOTTIP IncRNA Promotes Hematopoietic Stem Cell Self-Renewal Leading to AML-like Disease in Mice, Cancer Cell, 36 (2019) 645-659 e648.

[22] J.S. Mattick, RNA regulation: a new genetics?, Nat Rev Genet, 5 (2004) 316-323.

[23] F.Q. Nie, M. Sun, J.S. Yang, M. Xie, T.P. Xu, R. Xia, Y.W. Liu, X.H. Liu, E.B. Zhang, K.H. Lu, Y.Q. Shu, Long noncoding RNA ANRIL promotes non-small cell lung cancer cell proliferation and inhibits apoptosis by silencing KLF2 and P21 expression, Mol Cancer Ther, 14 (2015) 268-277.

[24] C.P. Ponting, P.L. Oliver, W. Reik, Evolution and functions of long noncoding RNAs, Cell, 136 (2009) 629-641.

[25] J.R. Prensner, W. Chen, M.K. lyer, Q. Cao, T. Ma, S. Han, A. Sahu, R. Malik, K. Wilder-Romans, N. Navone, C.J. Logothetis, J.C. Araujo, L.L. Pisters, A.K. Tewari, C.E. Canman, K.E. Knudsen, N. Kitabayashi, 
M.A. Rubin, F. Demichelis, T.S. Lawrence, A.M. Chinnaiyan, F.Y. Feng, PCAT-1, a long noncoding RNA, regulates BRCA2 and controls homologous recombination in cancer, Cancer Res, 74 (2014) 1651-1660.

[26] J. Przybilla, L. Hopp, M. Lubbert, M. Loeffler, J. Galle, Targeting DNA hypermethylation: Computational modeling of DNA demethylation treatment of acute myeloid leukemia, Epigenetics, 12 (2017) 886-896.

[27] J. Quintana, P. Advis, A. Becker, V. Beresi, M. Campbell, E.F. Vines, H. Garcia, C. Salgado, L. Vargas, J. Rojas, M. Obando, L. Neira, E. Paez, P. Zolezzi, Acute myelogenous leukemia in Chile PINDA protocols 87 and 92 results, Leukemia, 19 (2005) 2143-2146.

[28] T.C. Roberts, K.V. Morris, M.J. Wood, The role of long non-coding RNAs in neurodevelopment, brain function and neurological disease, Philos Trans R Soc Lond B Biol Sci, 369 (2014).

[29] M. Sauvageau, G. Sauvageau, Polycomb group proteins: multi-faceted regulators of somatic stem cells and cancer, Cell Stem Cell, 7 (2010) 299-313.

[30] L.Y. Sun, X.J. Li, Y.M. Sun, W. Huang, K. Fang, C. Han, Z.H. Chen, X.Q. Luo, Y.Q. Chen, W.T. Wang, LncRNA ANRIL regulates AML development through modulating the glucose metabolism pathway of AdipoR1/AMPK/SIRT1, Mol Cancer, 17 (2018) 127.

[31] S. Visser, X. Yang, LATS tumor suppressor: a new governor of cellular homeostasis, Cell Cycle, 9 (2010) 3892-3903.

[32] F. Wang, H. Yang, Z. Deng, Y. Su, Q. Fang, Z. Yin, HOX Antisense lincRNA HOXA-AS2 Promotes Tumorigenesis of Hepatocellular Carcinoma, Cell Physiol Biochem, 40 (2016) 287-296.

[33] J. Wang, Z. Su, S. Lu, W. Fu, Z. Liu, X. Jiang, S. Tai, LncRNA HOXA-AS2 and its molecular mechanisms in human cancer, Clinica chimica acta; international journal of clinical chemistry, 485 (2018) 229-233.

[34] K.C. Wang, H.Y. Chang, Molecular mechanisms of long noncoding RNAs, Mol Cell, 43 (2011) 904-914.

[35] Y. Wang, R. Zhang, G. Cheng, R. Xu, X. Han, Long non-coding RNA HOXA-AS2 promotes migration and invasion by acting as a ceRNA of miR-520c-3p in osteosarcoma cells, Cell Cycle, 17 (2018) 1637-1648.

[36] W. Wu, Y. Zhao, E. Gao, Y. Li, X. Guo, T. Zhao, W. He, H. Zhang, LncRNA DLEU2 accelerates the tumorigenesis and invasion of non-small cell lung cancer by sponging miR-30a-5p, J Cell Mol Med, 24 (2020) 441-450.

[37] M. Xie, M. Sun, Y.N. Zhu, R. Xia, Y.W. Liu, J. Ding, H.W. Ma, X.Z. He, Z.H. Zhang, Z.J. Liu, X.H. Liu, W. De, Long noncoding RNA HOXA-AS2 promotes gastric cancer proliferation by epigenetically silencing P21/PLK3/DDIT3 expression, Oncotarget, 6 (2015) 33587-33601. 
[38] X. Xue, Y.A. Yang, A. Zhang, K.W. Fong, J. Kim, B. Song, S. Li, J.C. Zhao, J. Yu, LncRNA HOTAIR enhances ER signaling and confers tamoxifen resistance in breast cancer, Oncogene, 35 (2016) 27462755.

[39] Y. Zhang, J. Xu, S. Zhang, J. An, J. Zhang, J. Huang, Y. Jin, HOXA-AS2 Promotes Proliferation and Induces Epithelial-Mesenchymal Transition via the miR-520c-3p/GPC3 Axis in Hepatocellular Carcinoma, Cell Physiol Biochem, 50 (2018) 2124-2138.

[40] H. Zhao, X. Zhang, J.B. Frazao, A. Condino-Neto, P.E. Newburger, HOX antisense lincRNA HOXA-AS2 is an apoptosis repressor in all trans retinoic acid treated NB4 promyelocytic leukemia cells, J Cell Biochem, 114 (2013) 2375-2383.

\section{Tables}

Table 1. Sequences of primers for qRT-PCR and siRNA sequence

\begin{tabular}{|ll|}
\hline Name & Sequences $\left(\mathbf{5}^{\prime}\right.$ to 3 $^{\prime}$ ) \\
\hline Primers for qRT-PCR & \\
\hline HOXA-AS2 (Forward) & CCCGTAGGAAGACCGATGA \\
\hline HOXA-AS2 (Reverse) & TTTAGGCCTTCGCAGACAGC \\
\hline GAPDH (Forward) & AGAAGGCTGGGGCTCATTTG \\
\hline GAPDH (Reverse) & AGGGGCCATCCACAGTCTTC \\
\hline LATS2 (Forward) & ACCCCAAAGTTCGGACCTTAT \\
\hline LATS2 (Reverse) & CATTTGCCGGTTCACTTCTGC \\
\hline EZH2 (Forward) & TGCACATCCTGACTTCTGTG \\
\hline EZH2 (Reverse) & AAGGGCATTCACCAACTCC \\
\hline Interference sequences (siRNA) & \\
\hline siHOXA-AS2 \#1 & GAGUUCAGCUCAAGUUGAACAUACA \\
\hline siHOXA-AS2 \#2 & AAACCUUGUAGAUAGCUUGAGCUGG \\
\hline siNC & UUCUCCGAACGUGUCACGUTT \\
\hline siEZH2 \#1 & GAGGUUCAGACGAGCUGAUUU \\
\hline siEZH2 \#2 & AAGACTCTGAATGCAGTTGCT \\
\hline
\end{tabular}

\section{Figures}


A

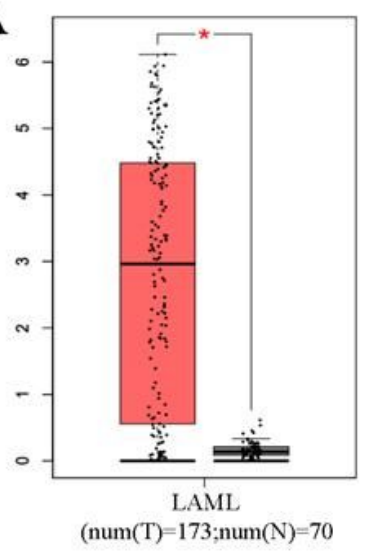

B

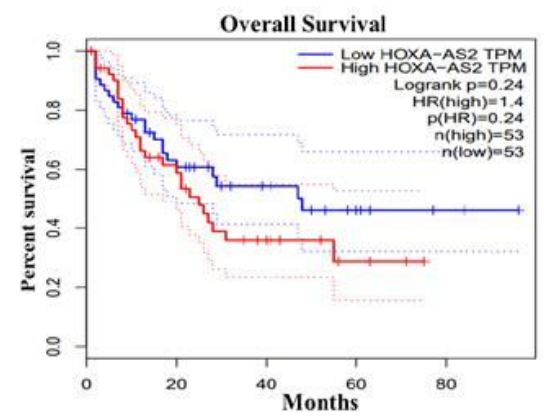

$\mathbf{E}$

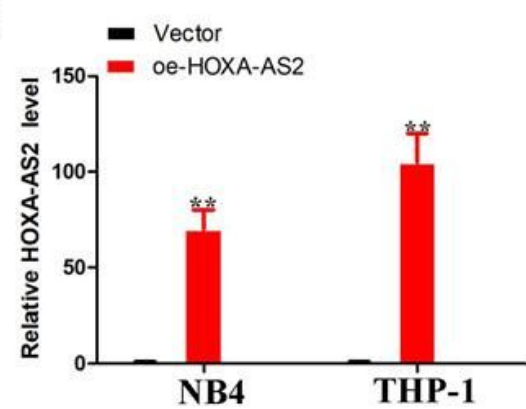

\section{Figure 1}

Relative expression of HOXA-AS2 in AML tissues, cells and its clinical significance. a Box plots for HOXAAS2 gene expression in AML and normal tissues from GEPIA. b Kaplan-Meier survival plot of overall survival of AML patients in GEPIA, categorized according to HOXA-AS2 gene expression (high vs. low, based on mean expression). c Relative expression levels in leukemia cell lines and SC cells. $d$ Relative expression of HOXA-AS2 in NB4 and THP-1 cells transfected with HOXA-AS2 plasmid. e Relative expression of HOXA-AS2 in NB4 and THP-1 cells transfected with siRNAs. ${ }^{*} p<0.05,{ }^{* \star} p<0.01$ 
A

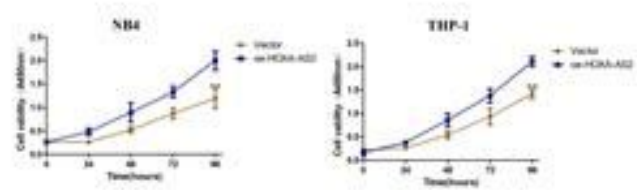

C

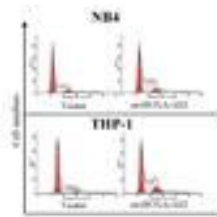

E

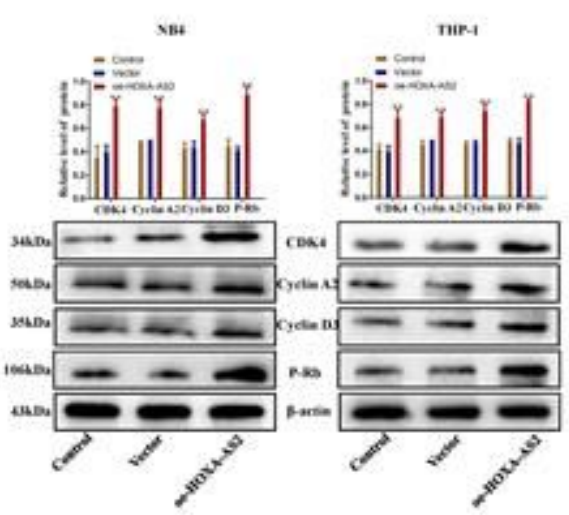

G

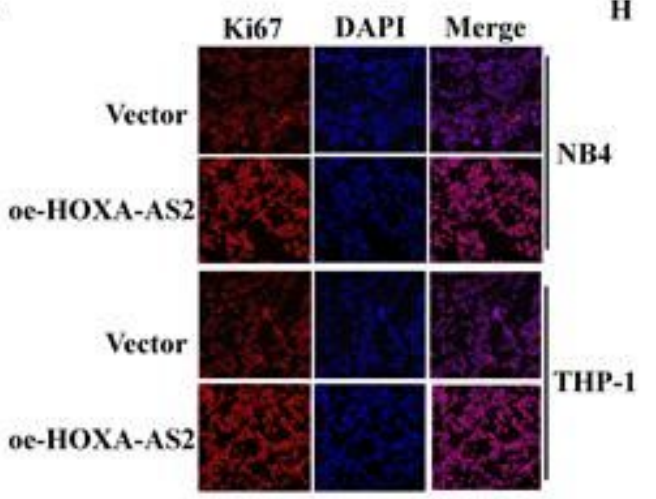

$\mathbf{F}$
B

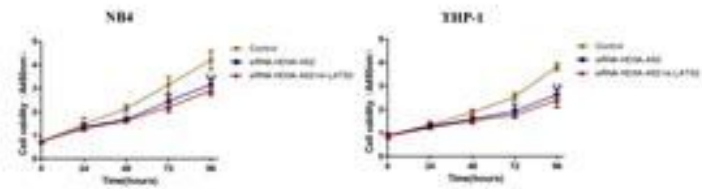

D
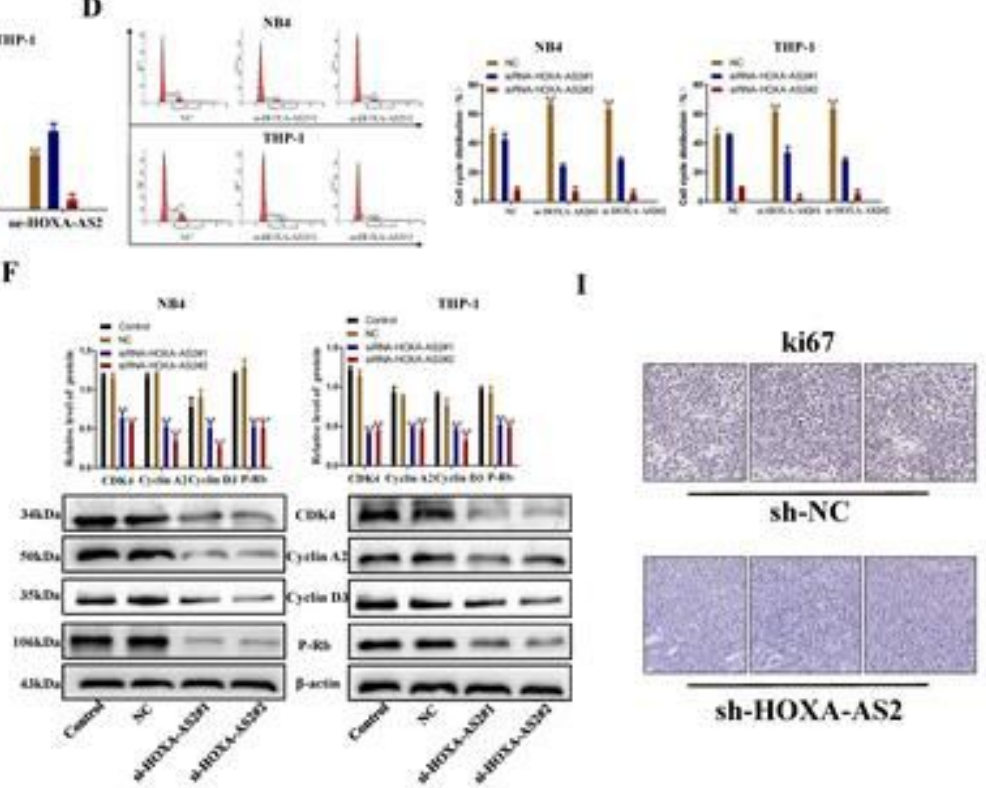

sh-HOXA-AS2

THP-1

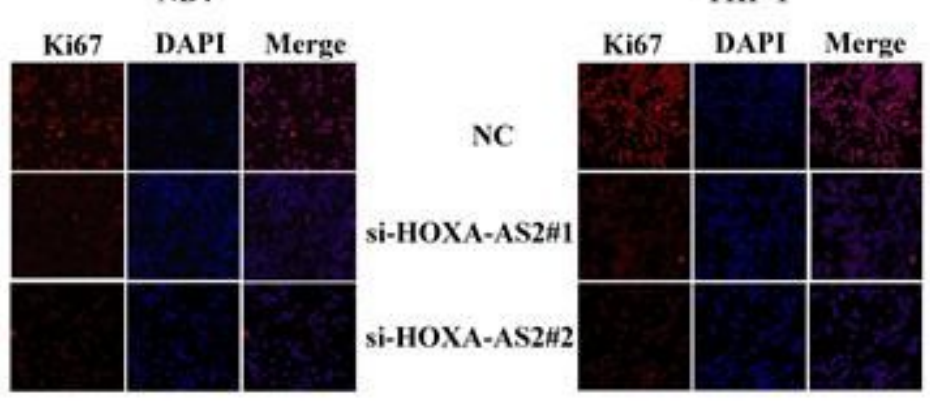

\section{Figure 2}

Effect of HOXA-AS2 on AML cells growth. $a, b$ The proliferation ability of NB4 and THP-1 cells transfected with HOXA-AS2 plasmid or transfected with si-HOXA-AS2\#1, si-HOXA-AS2\#2 was determined by CCK8 assays. $c$, $d$ The cell cycle of transfected NB4 and THP-1 cells. e, $f$ The levels of Cyclin D3, Cyclin A2, P-rb, and CDK4 protein in NB4 and THP-1 cells transfected with HOXA-AS2 plasmid or transfected with siHOXA-AS2\#1, si-HOXA-AS2\#2 were detected by western blotting assays. $\mathrm{g}$, $\mathrm{h}$ The level of ki67 protein in NB4 and THP-1 cells transfected with HOXA-AS2 plasmid or transfected with si-HOXA-AS2\#1, si-HOXAAS2\#2 was detected by immunofluorescence. i The ki67 expression of tumors from sh-HOXA-AS2 and shNC groups was determined by immunohistochemical staining. ${ }^{*} p<0.05, * * p<0.01$ 
A

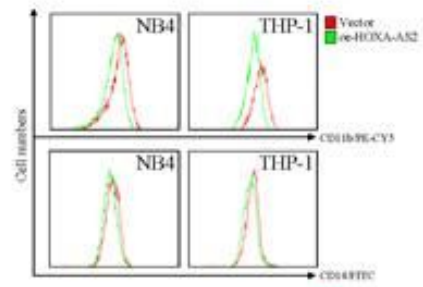

B

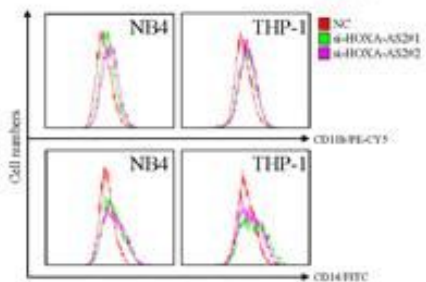

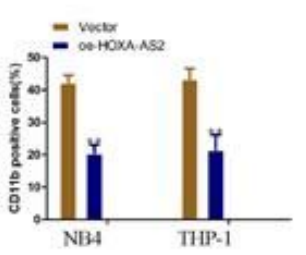

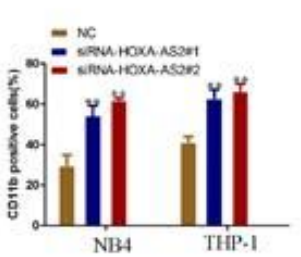

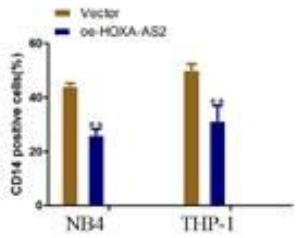



$\mathbf{E}$

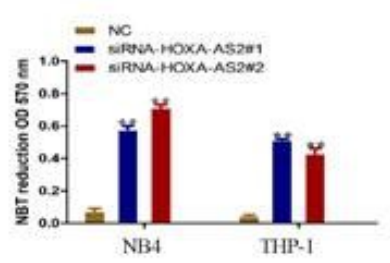

$\mathbf{F}$

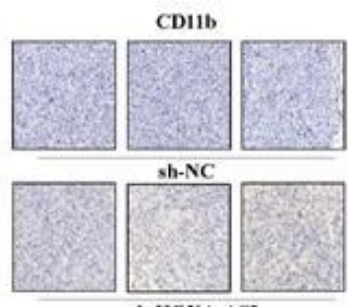

sh-HOXA-AS2

CD14
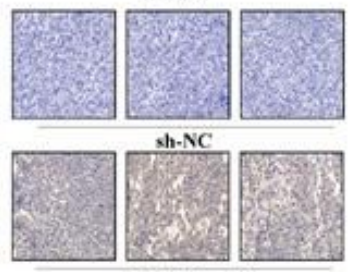

sh-HOXA-AS2

NB4
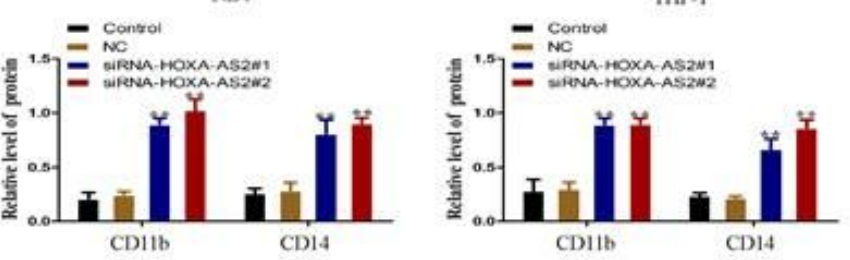

\section{Figure 3}

Effect of HOXA-AS2 on AML cells differentiation. a, b The differentiation ability of NB4 and THP-1 cells transfected with HOXA-AS2 plasmid or transfected with si-HOXA-AS2\#1, si-HOXA-AS2\#2 was determined by flow cytometry analysis. $c$, $d$ The levels of CD11b and CD14 protein in NB4 and THP-1 cells transfected with HOXA-AS2 plasmid or transfected with si-HOXA-AS2\#1, si-HOXA-AS2\#2 were detected by western blotting assays. e The differentiation ability of NB4 and THP-1 cells transfected with si-HOXAAS2\#1, si-HOXA-AS2\#2 was assessed by evaluating NBT reduction ability. $f$ The CD11b and CD14 expression of tumors from sh-HOXA-AS2 and sh-NC groups was determined by immunohistochemical staining. ${ }^{*} p<0.05,{ }^{* *} p<0.01$ 
A

NB4

THP-1
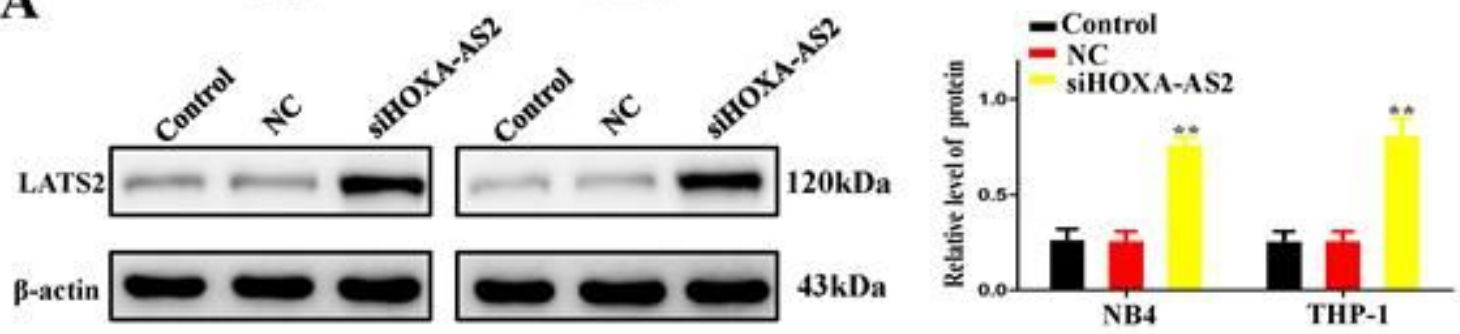

NB4

THP-1

B


C

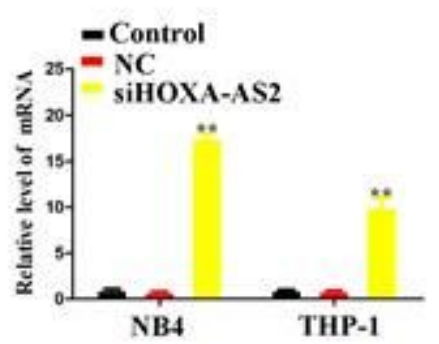

D

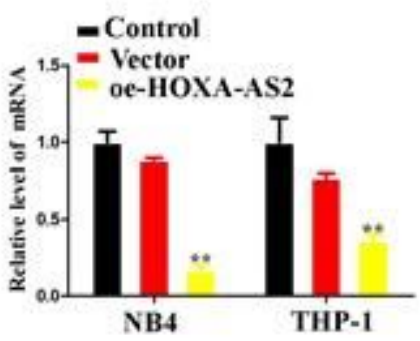

E

F
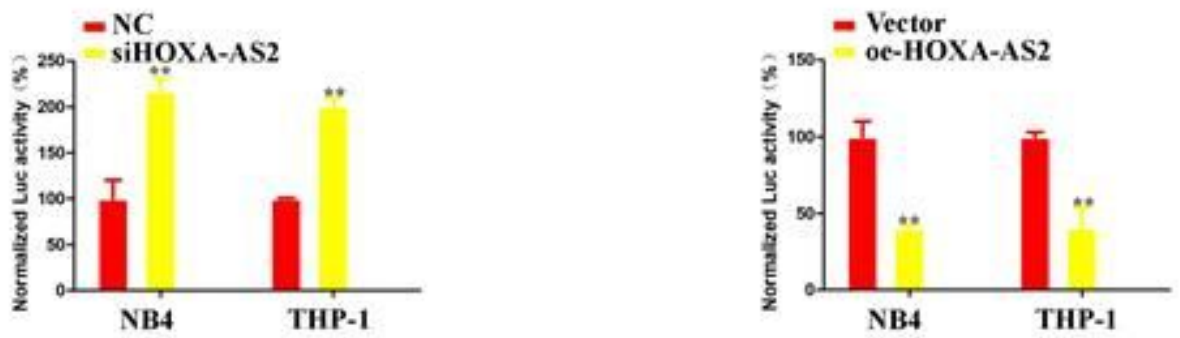

Figure 4

Upregulation of HOXA-AS2 inhibited LATS2 transcription in AML cells. $a$, b The LATS2 protein level was determined by western blot in NB4 and THP-1 cells transfected with HOXA-AS2 plasmid or transfected with si-HOXA-AS2\#1, si-HOXA-AS2\#2. c, d The LATS2 mRNA level was determined by qRT-PCR in NB4 and THP-1 cells transfected with HOXA-AS2 plasmid or transfected with si-HOXA-AS2\#1, si-HOXA-AS2\#2. e, $f$ LATS2 transcriptional activities of NB4 and THP-1 cells transfected with HOXA-AS2 plasmid or transfected with si-HOXA-AS2\#1, si-HOXA-AS2\#2. *p $<0.05,{ }^{* *} p<0.01$ 
$\mathbf{A}$
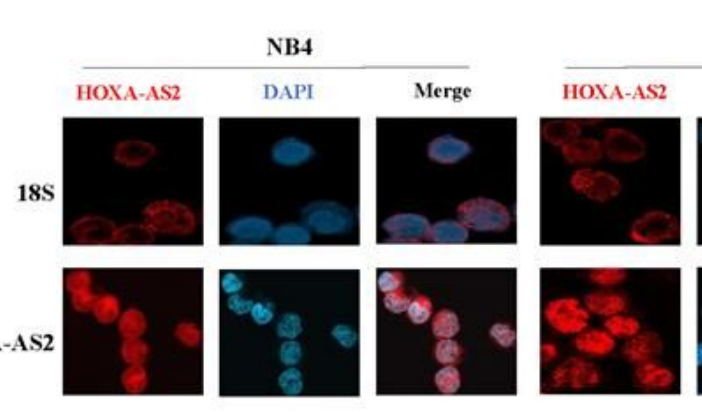

THP-1
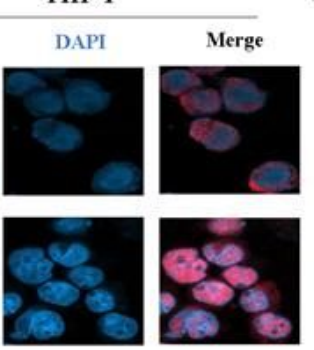

B

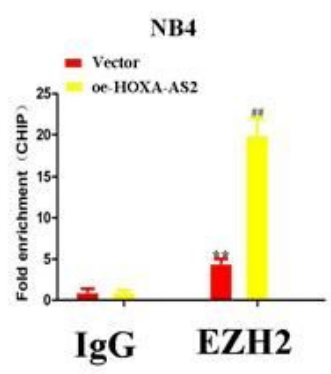

THP-1

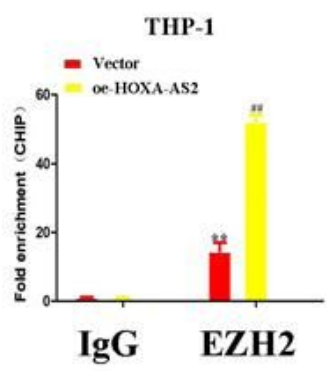

C

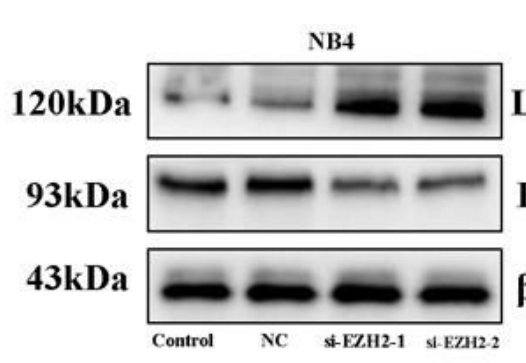

D

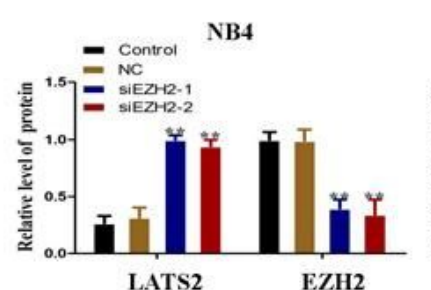

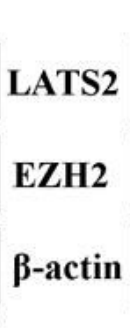

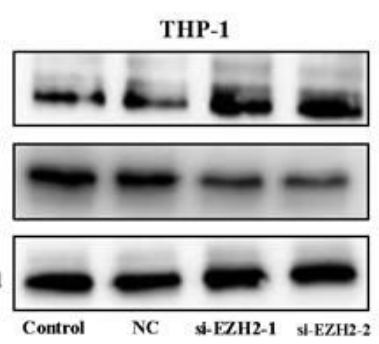

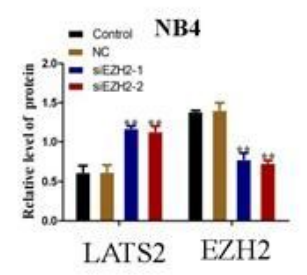

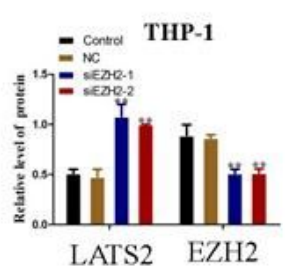

F

$\mathbf{E}$
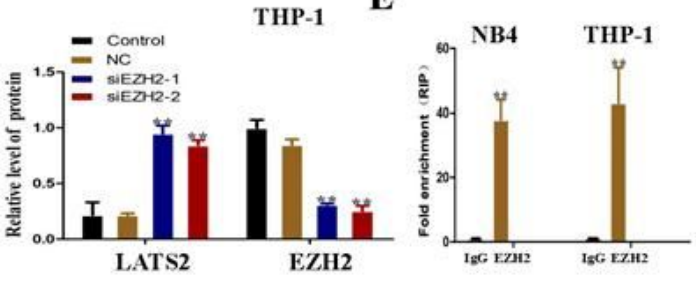

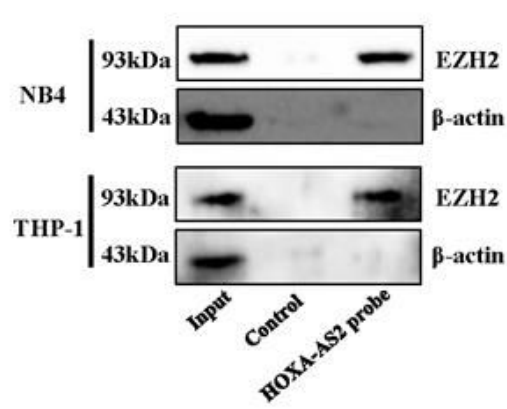

Figure 5

HOXA-AS2 downregulated transcriptional expression of LATS2 through recruitment of EZH2 in AML cells. a HOXA-AS2 distribution in NB4 and THP-1 cells was detected by FISH. b HOXA-AS2 plasmid was transfected into NB4 and THP-1 cells, and chrome immunoprecipitations were performed by using specific anti-EZH2 antibodies. c NB4 and THP-1 cells transfected with EZH2 siRNAs or the control siRNA for $72 \mathrm{~h}$ were collected, and EZH2 and LATS2 protein levels were detected by Western-blot assay. $\mathrm{d}$ EZH2 siRNAs $(E Z H 2-1,2)$ or the control siRNA were transfected into NB4 and THP-1 cells for $48 \mathrm{~h}, \mathrm{EZH} 2$ and LATS2 mRNA levels were then assessed by RT-qPCR. e RNA immunoprecipitations were performed in NB4 and THP-1 cells, and the relative quantities of HOXA-AS2 were detected by RT-qPCR assay, normalized to the input groups. IgG and EZH2 represented for the groups coprecipitation with IgG protein and antiEZH2 antibody respectively. $f$ Total proteins were extracted from NB4 and THP- 1 cells, and then HOXAAS2 pull-down assay was performed. The EZH2 protein levels were evaluated by Western-blot. HOXA-AS2 probe represented the biotin-labeled HOXA-AS2 probe group and control stood for the oligo probe group. ${ }^{*} p<0.05,{ }^{* *} p<0.01$ 
A

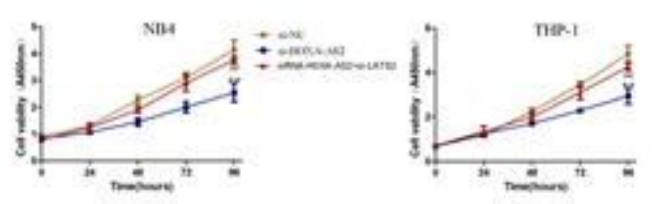

B
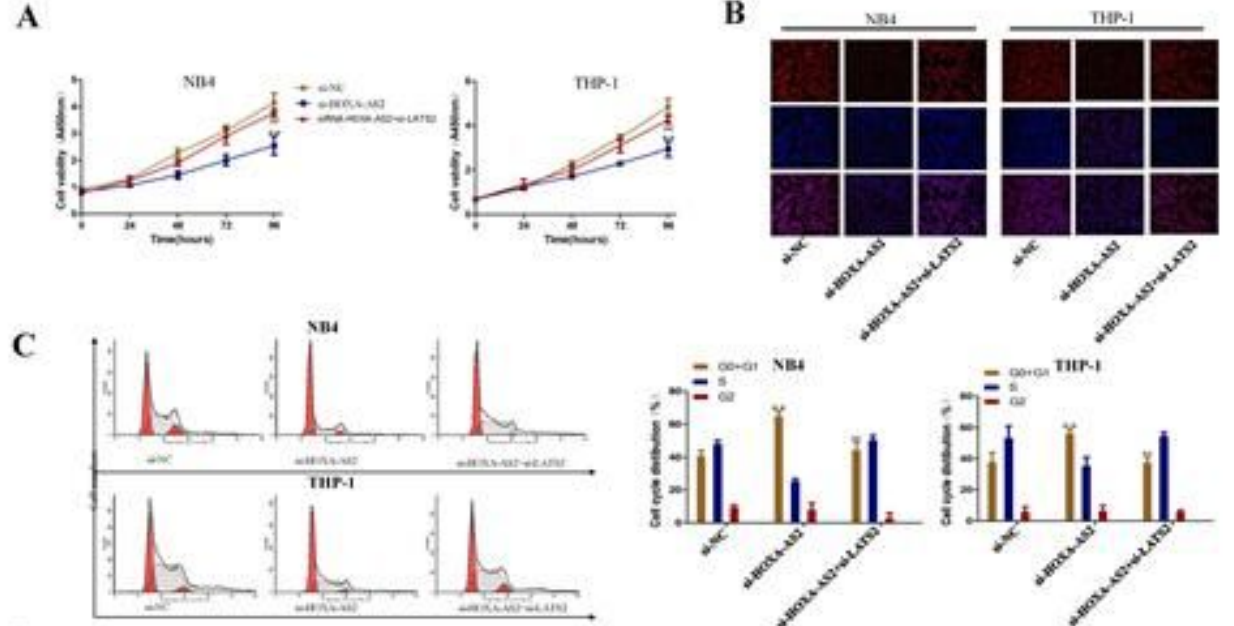

D
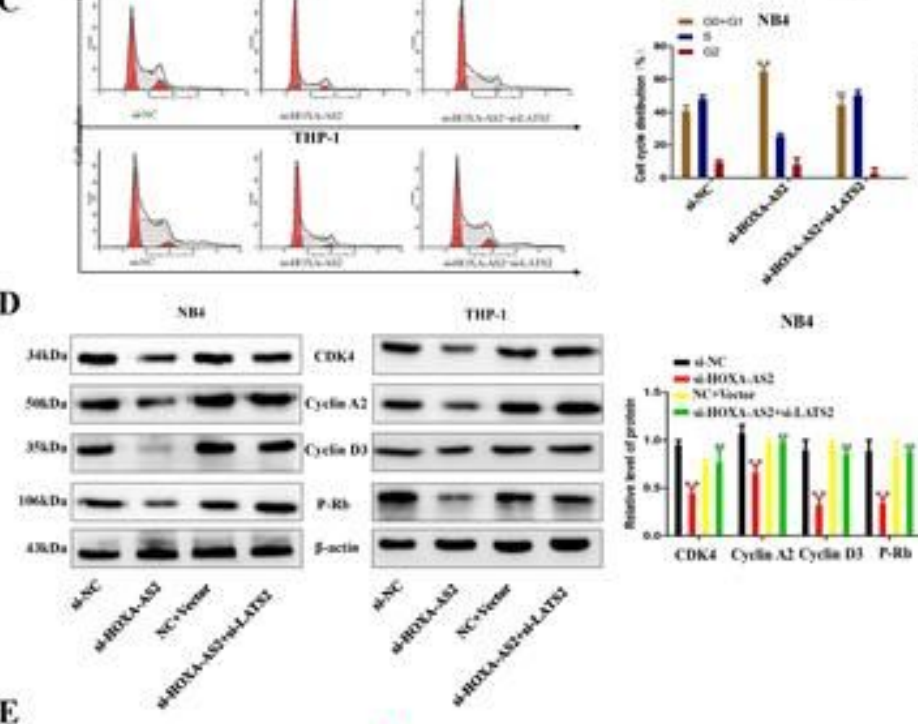

NBA

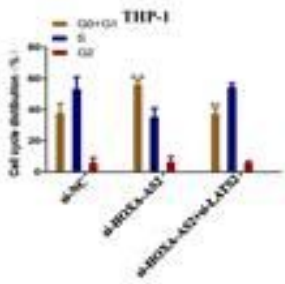

IIIP-1
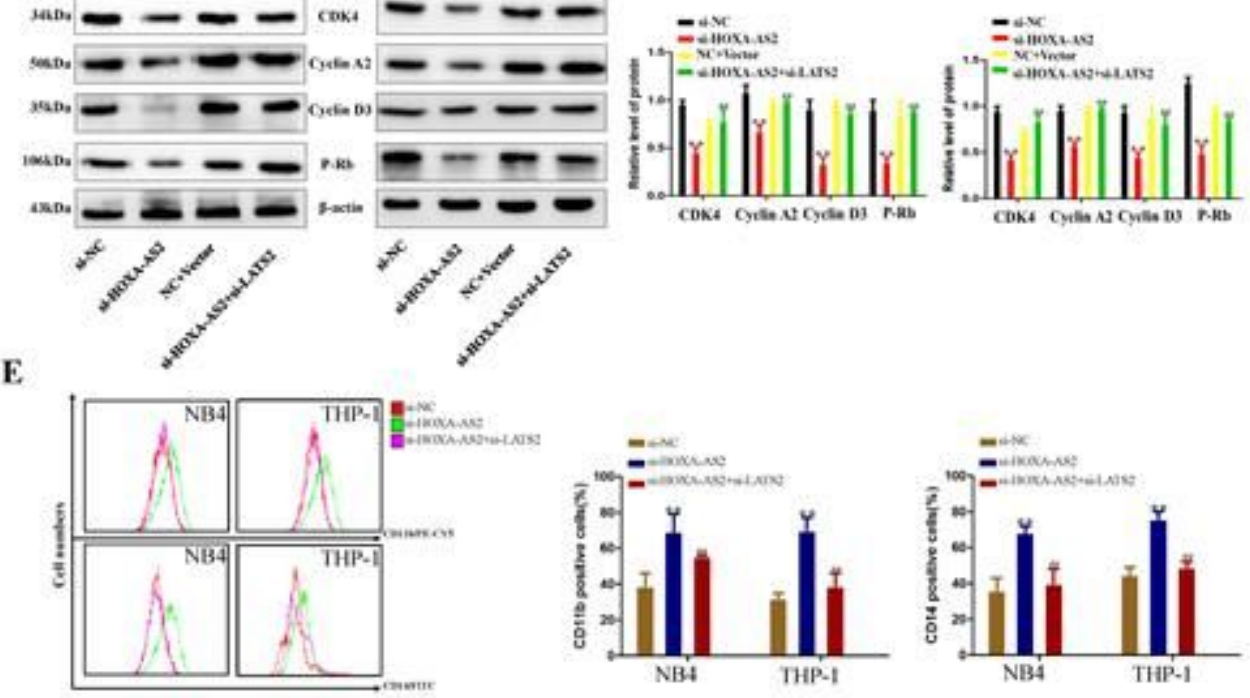

F

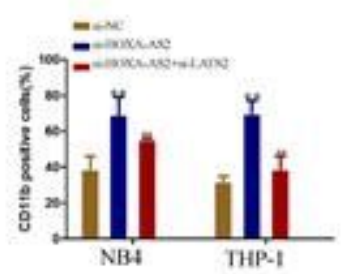

NB4
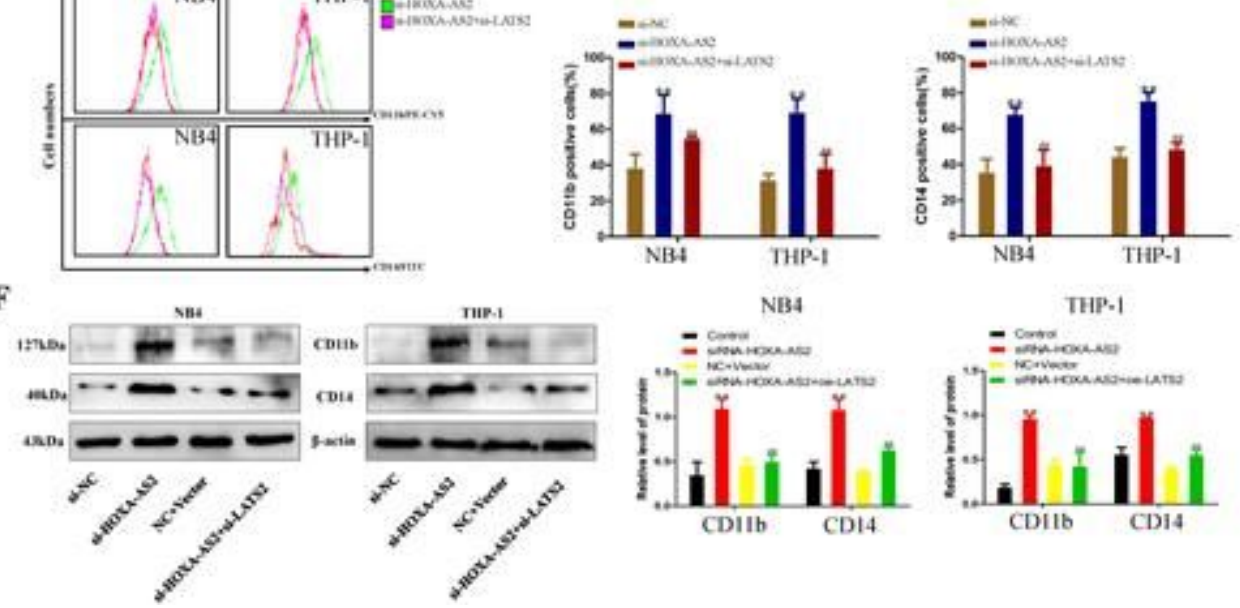

THP.1

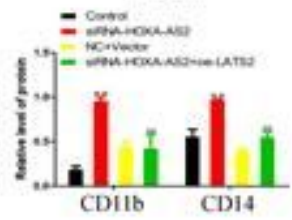

\section{Figure 6}

Silencing LATS2 potentially involves the oncogenic function of HOXA-AS2. a CCK8 assay was performed to determine the cell viability for si-HOXA-AS2 and si-LATS2 co-transfected NB4 and THP-1 cells. b The cell cycle of transfected NB4 and THP-1 cells. c Western blot analysis of cyclin D3, cyclin A2, P-rb, and CDK4 after si-HOXA-AS2 and si-LATS2 co-transfection in NB4 and THP-1 cells. $\beta$-actin protein was used as an internal control. $d$ The level of ki67 protein in NB4 and THP-1 cells co-transfection with si-HOXAAS2 and si-LATS2 was detected by immunofluorescence. e The levels of CD11b and CD14 protein in NB4 and THP-1 cells co-transfection with si-HOXA-AS2 and si-LATS2 were detected by flow cytometry analysis. $f$ Western blot analysis of CD11b and CD14 after si-HOXA-AS2 and si-LATS2 co-transfection in 
NB4 and THP-1 cells. $\beta$-actin protein was used as an internal control. $* p<0.05, * * p<0.01$, compared with the NC siRNA group. \#p < 0.05, \#\#p <0.01, compared with the si-HOXA-AS2 group

A

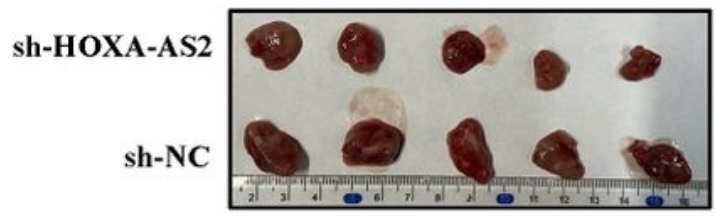

C

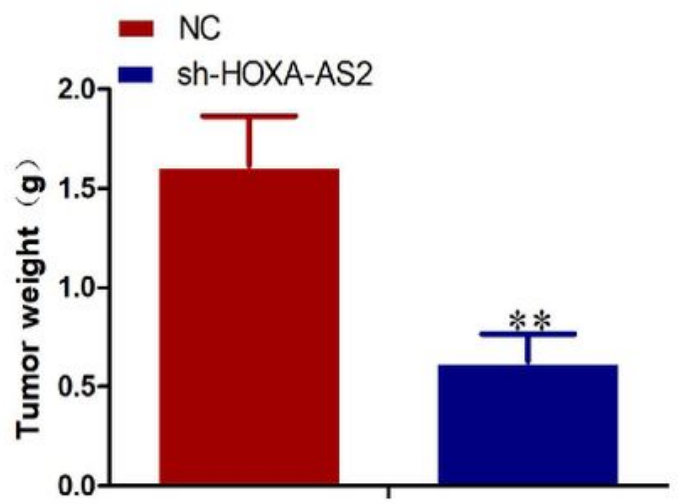

$\mathbf{E}$

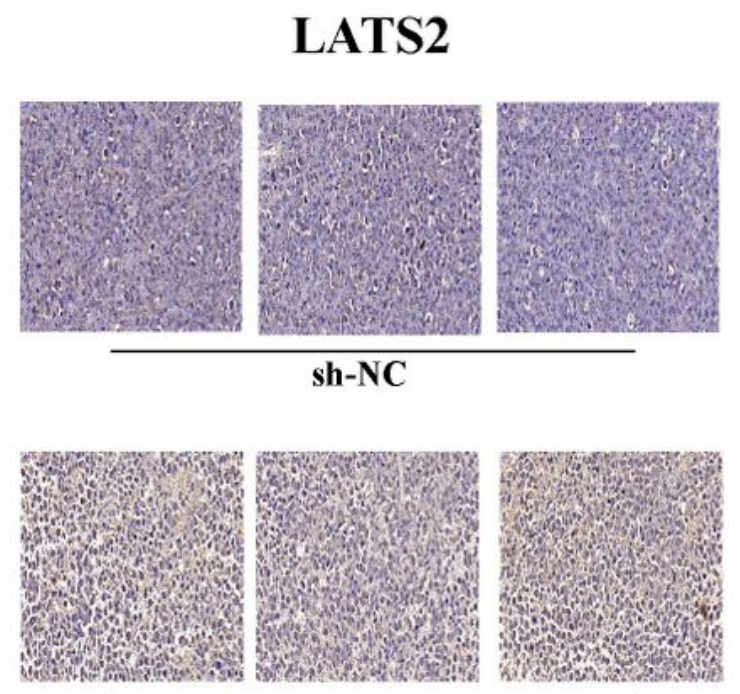

sh-HOXA-AS2
B

D
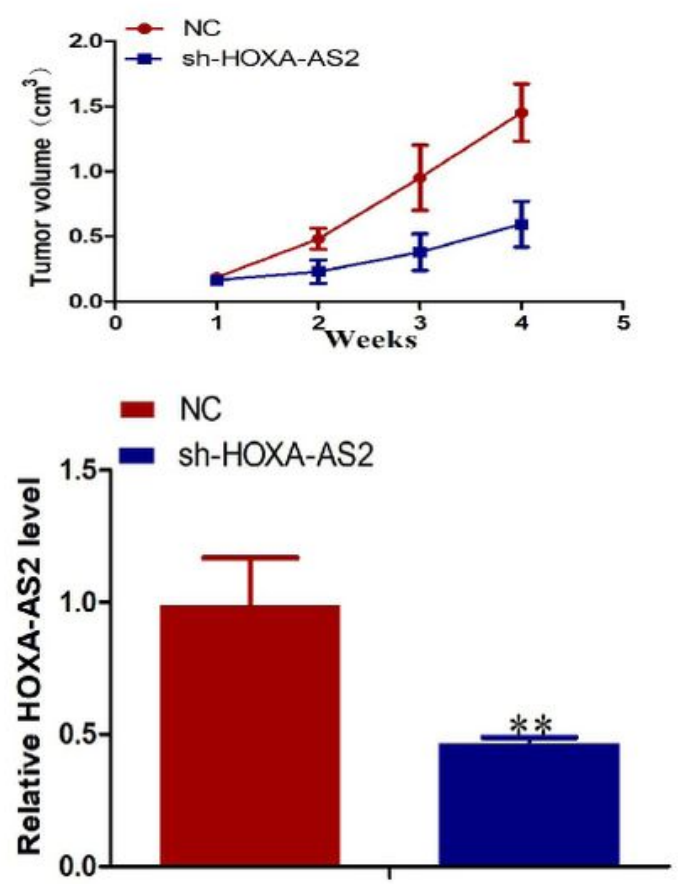

$\mathbf{F}$

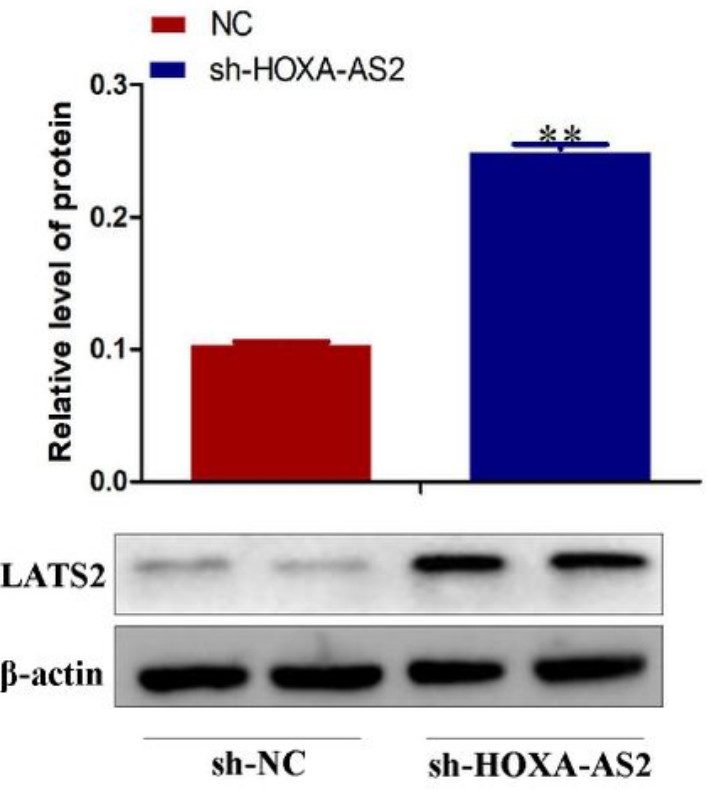

Figure 7

Knockdown of HOXA-AS2 inhibits AML tumorigenesis in vivo. a The total number of tumors after removal from the mice. $b$ The tumor volumes were calculated every week after inoculation. $c$ The tumor weights after the tumors were harvested. $d$ qRT-PCR analyses indicated that the HOXA-AS2 expression was 
significantly increased in vivo. e Then, protein was extracted from the tumor tissues, and expressions of LATS2 protein was detected by Western blot. $\mathrm{f} \mathrm{Immunohistochemistry} \mathrm{analysis} \mathrm{of} \mathrm{LATS2} \mathrm{was} \mathrm{obtained}$ from tumors. $\beta$-actin served as a loading control. $* p<0.05, * * p<0.01$, compared with the NC shRNA group. 